\title{
Vascular Bifurcation Detection in Scale-Space
}

\author{
Daniel-Marian Baboiu and Ghassan Hamarneh \\ Simon Fraser University \\ Burnaby BC, Canada, V5A 1S6 \\ dba28@alumni.sfu.ca
}

\begin{abstract}
Several methods have been proposed over the years for segmentation of vessels, many of them based on scalespace. However, none of the existing methods for blood vessel segmentation is appropriate for extension to bifurcation detection. Other existing bifurcation detection algorithms use an inherently serial "track and detect" approach, which also requires a seed point. We present for the first time a comprehensive scale-space analysis of vascular bifurcations, resulting in a simple, novel algorithm for direct detection of blood vessel bifurcation points based not only on spatial variation across scales, but also on the variation at a single spatial point across scales, without requiring training data or seed points. We present an analytical model for the bifurcation evolution with increasing scale, which was combined with eigenvalue analysis to create a bifurcation-Ness filter. We reveal, for the first time, a hybrid structure of bifurcations in scale-space. The algorithm was tested for validation in both $2 D$ and $3 D$, with synthetic data as well as medical and non-medical images.
\end{abstract}

\section{Introduction}

While significant progress has been made over the years in the segmentation of the vessels themselves, automatic detection of bifurcation points remains a significant difficulty. Several approaches have been proposed, for example based on centerline extraction [13] or on active models (snakes [11] and vessel crawlers [12]). However, the centerlinebased methods either do not handle bifurcations (in some cases because the response function tracked decreases at bifurcations) or have difficulties ensuring continuity at vessel junctions.

A comprehensive review [6] groups vessel extraction techniques in six families, including artificial intelligence approaches. These appear to be the most promising in terms of sensitivity and accuracy ([16] reports success rate of 97\%). However, methods based on artificial intelligence are computationally expensive and require training data, and even [16] uses a track-and-detect approach, requiring a seed. A more recent review [8] states that bifurcation detection is still one of the most challenging tasks in automated blood vessel segmentation, with only a handful of papers specifically dedicated to bifurcation detection. All these approaches are inherently serial, appropriate for computers with only a few cores, with parallelism used only for processing local data.

From an image feature point of view, blood vessels are ridges, and the corresponding descriptors can be used. Most notably, the scale-space Hessian analysis [3] results in a vesselness operator, based on Gaussian penalties for deviations from ideal values. This approach does not detect the bifurcation points; in fact, the vesselness measure defined drops significantly at bifurcation points (although not to 0 ), leading to disconnected vessel segments.

The approach we propose is a simple and direct method, based on the scale space behavior of bifurcations. This approach uses the scale-space transform not only to find the maximum across scales, but also for extracting additional information from the rate of change across scales (the first scale-space approach to do so). It is intended to be used mainly as a "first line" filter for more complex, area-based filters, although its performance is good enough to be used by itself. Unlike the the serial approach of track-and-detect methods, the filter we propose processes the whole image in parallel, and thus is able to take full advantage of modern massively multicore computer architectures. As a result of using scale-space - a smoothing of the initial image with a set of Gaussians with progressively increasing widths the method proposed is robust to relatively small amounts of noise. Only basic image processing techniques, such as background subtraction and contrast stretching, were used. Most of the tests of the algorithm were done with 2D images (synthetic as well as clinical), but the results are general, and the theoretical model is valid for volumetric data as well. There is no loss of generality in testing, since theoretical models of bifurcations show that the branches of the vessels in a bifurcation should lie in the same plane [5]. This is confirmed by an extensive experimental study made using 
vascular casts of the arterial system of a laboratory rat, the first one measuring the 3D structure of bifurcations [15].

\section{Scale-Space background and bifurcation model}

The concept of scale space was introduced in $[7,2]$. It comes from the intuitive concepts that each object in an image has limited extent ("outer scale") and a limited resolution ("inner scale"), which define the relevant range of scales. To obtain the scale-space representation of an image, the original image $L(\mathbf{x})$ is embedded in a one-parameter family of derived images $L(\mathbf{x}, \sigma)$ (where $\sigma$ is the scale) which are studied as a family. This family is found to be the convolution of the original image with isotropic Gaussian functions of different widths ("diffusion")

$$
L(\mathbf{x}, \sigma)=L(\mathbf{x}) \star G(\mathbf{x}, \sigma)
$$

where the D-dimensional isotropic Gaussian is defined as

$$
G(\mathbf{x}, \sigma)=\frac{1}{\sqrt{2 \pi \sigma^{2}} D} e^{-\frac{\|\mathbf{x}\|^{2}}{2 \sigma^{2}}}
$$

Natural coordinates at scale level $s$ are defined as the dimensionless frequency $\boldsymbol{\Omega}=\sigma \omega$ (with $\omega$ being the usual spatial frequency), or equivalently as the dimensionless spatial coordinate $\mathbf{X}=\mathbf{x} / \sigma$. Differential operators are defined in these "natural coordinates". Due to the properties of Gaussians, derivatives of the image in scale-space are convolutions of the original image with the corresponding $n$-th order partial derivatives of the Gaussian kernel,

$$
G_{i_{1}, \ldots, i_{n}} \rightarrow \sigma^{\gamma n} \partial_{i_{1}, \ldots, i_{n}} G_{i_{1}, \ldots, i_{n}}
$$

where the subscripts $i_{1}, i_{2} \ldots i_{n}$ are used to represent partial derivatives with respect to $i_{j}$-th spatial coordinate. The parameter $\gamma$ was introduced by Lindeberg [10] to properly identify the scale of various feature, described mathematically by differential operators. For detection of quasi-linear features like blood vessels, this parameter is 1 .

Since blood vessels are bright structures, their centerline is a line of maximum intensity; the first derivative (the gradient of the image intensity) is 0 , so all the relevant information is encoded in the matrix of second-order derivatives the Hessian. This is a second-order symmetric tensor, with the geometric interpretation as an ellipsoid oriented along the vessel. The size of the eigenvalues is used to extract information about how close is the structure to a vessel; for details, see [3]. Here we use the same convention, with $\lambda_{1}$ the smallest eigenvalue (in magnitude) and $\lambda_{3}$ the largest.

\subsection{Vessel and bifurcation response in scale-space}

The basis of the approach we propose is the response of a straight semi-infinite ideal vessel. The geometry is illustrated in Figure 1(a). The vessel has a finite transverse extension (in the $y$ coordinate) of radius $w$; in this direction,

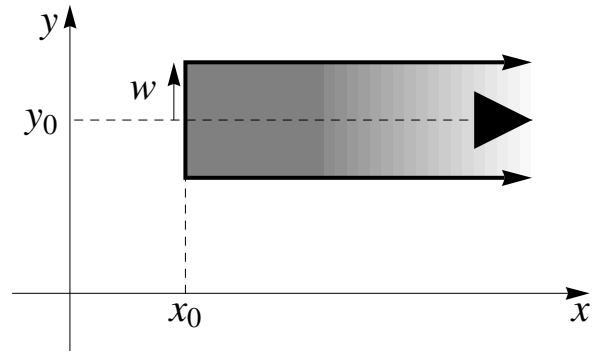

(a)

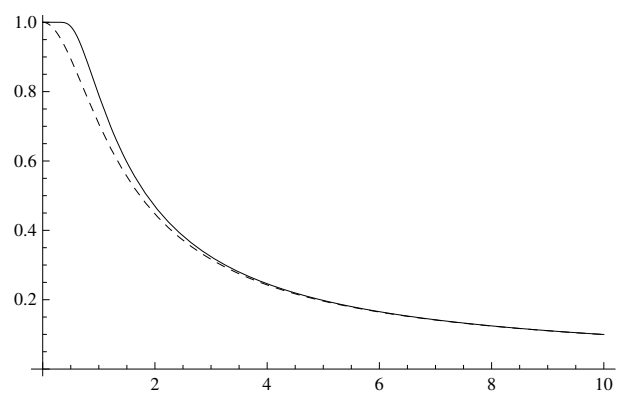

(b)

Figure 1. (a) The geometry for calculating scale-space response of a semi-infinite vessel with a radius $w$, at distance $y_{0}$ from the axis of the vessel and offset $x_{0}$ from its end. (b) Decay of intensity at the center of vessel with scale (on horizontal scale), for rectangular profile (continuous line) and Gaussian profile (dashed line) with width $w=1$.

the profile can be rectangular or Gaussian, two profiles for which the results can be obtained in closed form. The vessel segment starts at $x_{0}$ and continues to infinity, with uniform profile. This idealization allows fully analytical calculations to be made using separation of variables. For a vessel with a Gaussian profile, the limits in $y$, as shown in Figure 1(a), represent the standard deviation of the profile. Such a vessel with Gaussian profile has the scale-space response

$L\left(x_{0}, y_{0} ; \sigma\right)=\int_{-\infty}^{\infty}\left(\int_{x_{0}}^{\infty} \frac{1}{2 \pi \sigma^{2}} e^{-\frac{x^{2}+y^{2}}{2 \sigma^{2}}} e^{-\frac{\left(y-y_{0}\right)^{2}}{2 w^{2}}} d x\right) d y$

This integral evaluates to

$L\left(x_{0}, y_{0} ; \sigma\right)=\frac{w}{2 \sqrt{\sigma^{2}+w^{2}}} e^{-\frac{y_{0}^{2}}{2\left(\sigma^{2}+w^{2}\right)}}\left[1-\operatorname{Erf}\left(\frac{x_{0}}{\sigma \sqrt{2}}\right)\right]$

An important feature of the scale-space is that once the scale goes above the width of the vessel, typically $\sigma$ above $1.5-2$ vessel radii $w$, all influence of the internal structure of the vessel practically vanishes. Vessels with a different intensity profile will have an intensity decay governed by the same law, with a profile-dependent effective width. This is consistent with using a scale above the "inner scale", where the inner structure of the vessel is irrelevant. While 
intuition supports the idea that vessel structure elements are mixed together by convolution with a wide Gaussian, a rigorous proof can be made using the decomposition of the vessel profile using Hermite functions [14]. The fundamental mode is the Gaussian profile, while the higher harmonics are obtained from derivatives of the Gaussian and have higher decay rates with scale. On the axis of the vessel, $y_{0}=0$ and the dominant term of the intensity at large scale $(\sigma \gg w)$ is $w / \sqrt{w^{2}+\sigma^{2}}$, with $w$ being the effective width of the vessel. In practice, this approximates well the intensity decay with increasing scale for $\sigma \gtrsim 2 w$, as can be seen in Figure 1(b)

In geometries with higher number of dimensions, the calculations are similar. For 3D vessels, there is an additional factor in the third coordinate $z$ similar to the factor in $y$. The integrals can be calculated easily by separation of variables, resulting in a $w / \sqrt{w^{2}+\sigma^{2}}$ term for each transverse dimension. Thus, the dominant term of the decay rate is

$$
I(w, \sigma)=I_{0}\left(\frac{w}{\sqrt{w^{2}+\sigma^{2}}}\right)^{\delta}
$$

where $I(w, \sigma)$ is the intensity on the center of the vessel, as a function of the vessel width $w$ and the scale $\sigma$. Here, $\delta=1$ for a vessel in a 2D image (a line), as well as a sheet in $3 \mathrm{D}$, as these can diffuse in only one transverse dimension; $\delta=2$ for a "dot" in 2D (a blob), as well as a vessel in $3 \mathrm{D}$, and $\delta=3$ for a blob in 3D. This $\delta$, the number of transverse dimensions, is called codimension, and is number of dimensions in which diffusion can occur, excluding the diffusion internal to the diffused structure itself. We can calculate the codimension $\delta(\mathbf{x})$ at any point using the first $\left(I_{1}=(\partial I / \partial \sigma) / I\right)$ and second $\left(I_{2}=\left(\partial^{2} I / \partial \sigma^{2}\right) / I\right)$ normalized derivatives of the image intensity with respect to scale to obtain

$$
\delta=\frac{2 \sigma I_{1}^{2}}{\left(I_{2}-I_{1}^{2}\right) \sigma-I_{1}}
$$

This expression depends on Equation 6 not having an image background term, thus requiring background elimination in the preprocessing step. The codimension has the ideal value only for scales between the inner scale and the outer scale. For practical purposes, we employ the usual scale selection mechanism described in [10] by using the scale at which the filter response is maximal (to which we add $25 \%$, to allow the profile to get closer to the ideal). Then fit locally the image intensity variation with scale according to Eq. 6, considering $\delta$ a parameter to be determined. We will use the value of this codimension to identify bifurcations from other localized structures. To account for deviations from the model, as well as the presence of residual background, we allow the codimension to be within 0.5 of the ideal value.

A bifurcation is the linear superposition of three semiinfinite vessels. The bifurcation location is the intersection of three vessel centerlines; there, each vessel will contribute a term the form in Eq.6 to signal intensity. At scales large enough, the denominator is dominated by the scale and factors out, so the codimension of the bifurcation is the same as the codimension of the vessels forming it.

\section{Implementation of the Bifurcation Detector}

The bifurcation detector has two main components: a local feature detector and the codimension measure. One might think that classical detectors like Harris and ShiTomasi might work, but these are designed to work at the boundary between bright and dark areas. In [4], the curvature of level curves was proposed as indicator of potential bifurcations, but the figures show that this filter generates too many false positives while many true bifurcations remain undetected. It is more appropriate to look at the blob detectors, the Laplacian and Determinant of Hessian (DoH), which have the advantage of being easy to calculate and are invariant to rotations. Of these, the Laplacian has strong response not only at bifurcations, but also along the vessels. It is not very selective, therefore inappropriate for use. The $\mathrm{DoH}$, however, has very good localization properties. In the principal coordinate system, the Hessian becomes diagonal and is the product of eigenvalues; its localization properties come from the factor of $\lambda_{1}$, the smallest (in magnitude) eigenvalue. In all cases, the maximum is taken across all scales. A detailed analysis and comparison of the various can be found in [1].

It is very intuitive to understand why the smallest eigenvalue describes localized features - it is related to strong variation in all directions. In $3 \mathrm{D}$, vessel bifurcations have an additional property: they are mostly confined on one plane [15]. This indicates that they might also have a significant plate tensor component, described mathematically by $\left|\lambda_{3}\right|-\left|\lambda_{2}\right|$, the difference of the two largest eigenvalues. Our investigation (not included here) shows that a filter using Gaussian penalties (similar to Frangi "vesselness" [3]) does not give significant response at bifurcations, and that their nonlinear behavior, especially when multiple penalties are combined, may generate multiple local maxima around the desired feature (see the split response of the Frangi-style filter in Figure 2. Thus, in 3D, the simpler filters $\left|\lambda_{1}\right|,\left|\lambda_{1}\right|\left(\left|\lambda_{3}\right|-\left|\lambda_{2}\right|\right)$ and $\mathrm{DoH}$ give the best localization of the bifurcations. These operators, however, give strong responses in all places with strong second-order derivatives, including blobs and end-of-vessels. Of these, $\left|\lambda_{1}\right|\left(\left|\lambda_{3}\right|-\left|\lambda_{2}\right|\right)$ has the added advantage of eliminating the end-of-vessels, but is applicable only in 3D images. Our tests show that the main difference between these for detecting actual bifurcations is only in the sharpness around the local maximum.

The bifurcations are detected by using local maxima of $\left|\lambda_{1}\right|$ (2D) or $\left|\lambda_{1}\right|\left(\left|\lambda_{3}\right|-\left|\lambda_{2}\right|\right)$ (in 3D) (subject to the con- 


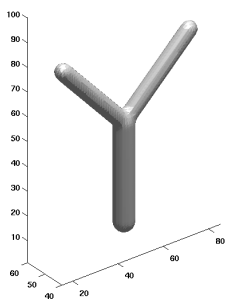

(a) Initial image, 3D

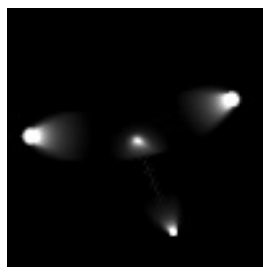

(d) $\left|\lambda_{1}\right|$

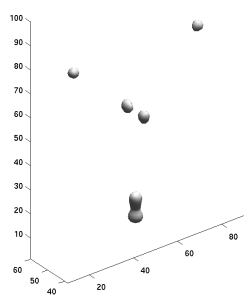

(b) Frangi-style, 3D

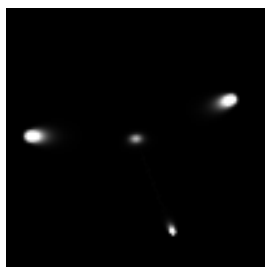

(e) $\mathrm{DoH}$

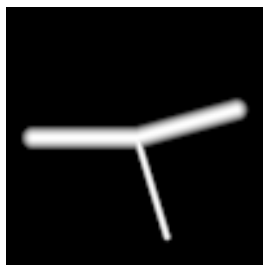

(c) Initial image, section

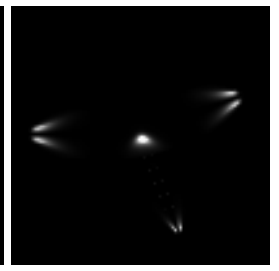

(f) $\left|\lambda_{1}\right|\left(\left|\lambda_{3}\right|-\left|\lambda_{2}\right|\right)$
Figure 2. Symmetric bifurcation (a) and response to Frangi-style filter (b), showing the split-maximum at bifurcation; Section through asymmetric bifurcation (c) and its responses to bifurcation filters: $\left|\lambda_{1}\right|(\mathrm{d}), \operatorname{DoH}(\mathrm{e})$, and $\left|\lambda_{1}\right|\left(\left|\lambda_{3}\right|-\left|\lambda_{2}\right|\right)$ (f).

straint for local intensity maxima, all Hessian eigenvalues must be negative), then are separated from other local features like blobs using the value of codimension. Other local maxima in the maps of these operators can be generated by noise and pixellation effects, but they have a characteristic dimension of one pixel and decay very quickly with increasing scale.

\section{Results}

To quantitatively evaluate the performance of the algorithm, we generated synthetic 2D bifurcations, with main branch radii $r_{0}$ from 1 to 7 pixels (in steps of 0.5 ) and bifurcation ratios $\alpha_{R}$ (ratios of the daughter vessels) from 0.1 to 1 (in steps of 0.05). These ranges are extreme; low values for $r_{0}$ and $\alpha_{R}$ lead to heavy pixelation of the smaller daughter vessel. Where global statistics were required, we used $r_{0}>=2$ (since real vessels do not present interruptions due to pixelation) and $\alpha_{0}>=0.3$, (corresponding to the smallest bifurcation area ratio of 0.1 reported experimentally [15]). To test noise robustness, we added Gaussian noise (with standard deviation between 0 and 1) and saltand-pepper noise with various densities. The images were generated with different orientations of the main branch, each with a different instance of noise, with a total of 180 runs for each set (noise, $r_{0}, \alpha_{R}$ ), using random orientations of the parent vessel. Bifurcation angles and branch radii were designed to mimic those of real bifurcations as described in [5, 15]. Some samples A mask with radius of 40 pixels was placed around the bifurcation, to isolate it and to avoid boundary artifacts. To limit spurious data, we imposed a limit on the saliency for the bifurcationness response of $10^{-4}$ of the maximum bifurcation response.

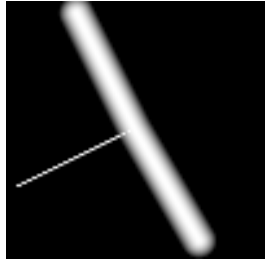

(a)

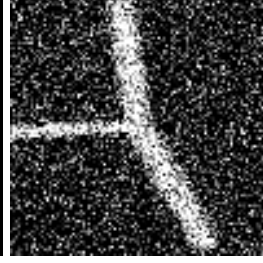

(b)

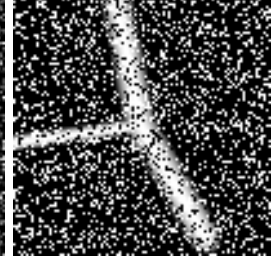

(c)
Figure 3. Typical bifurcations used in testing. First: $\alpha_{R}=0.1$, no noise; last two: $\alpha_{R}=0.5$, with Gaussian and Salt-and pepper noise respectively, with standard deviation and respectively density of 0.4

The statistics of this simulations are presented in Figure 4. Virtually all bifurcations were detected in the absence of the noise (with the exception of the extreme cases mentioned earlier). In the presence of noise, however, the probability of detection decreased; the highest noise level for which we achieved 95\% detection of true positives was standard deviation of 0.40 for Gaussian noise and 35\% density for the salt-and-pepper noise. A possible reason for this decrease is that the signal for the bifurcation itself might still be present, but with a value much lower than the threshold used, or that a strong noise pixel is present, and its codimension and scale dominate those of the bifurcation. Another reason is that high noise levels contribute to the effective background level, reducing the reliability of the codimension filter.

An important feature is the codimension selection filter. After applying the bifurcationness filter, there are up to 200 local maxima in the resulting map (in 2D images) and up to 8000 in 3D images; almost all of these are eliminated by the codimension filter and the scale selection. For example, in the case of salt-and-pepper noise, there maximum number of false positives (seen at high noise levels) is 3.31 per bifurcation if the scale threshold is set to 1 , and decreases to 0.71 if the scale threshold is increased to 1.5 and to 0.2 for scale threshold of 2. For Gaussian noise, the maximum values are 1.8, 0.33, and 0.1, for all noise levels (standard deviation up to 1). At such high noise levels, there is a significant risk of noise pixels to form clusters mimicking vessels and/or bifurcations. However, increasing the scale threshold increases the risk of false negatives. Since we intended this filter as a first round detector, to generate initial test points for more computationally expensive filters, it is better to keep the threshold at the low end; the false positives are eliminated in postprocessing with other algorithms.

Figure 5 shows the measured errors in bifurcation detection. We can see that there is a skew of measured errors with respect to the expected distance for a normal distribution of 


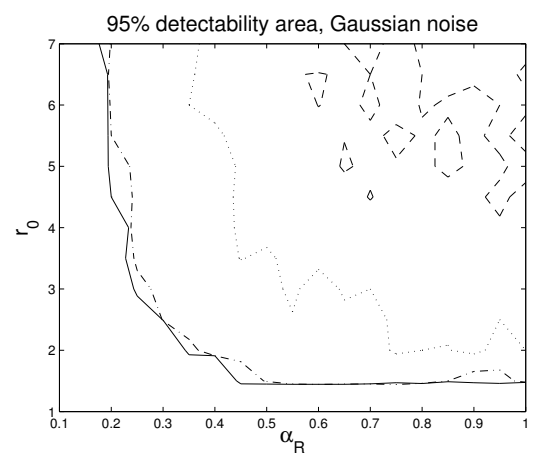

Figure 4. Areas of $95 \%$ detection probability (above the curves) for various radii of parent vessel $r_{0}$ and different bifurcation ratios $\alpha_{R}$ : Gaussian noise with standard deviation $0,0.1,0.25,0.40$

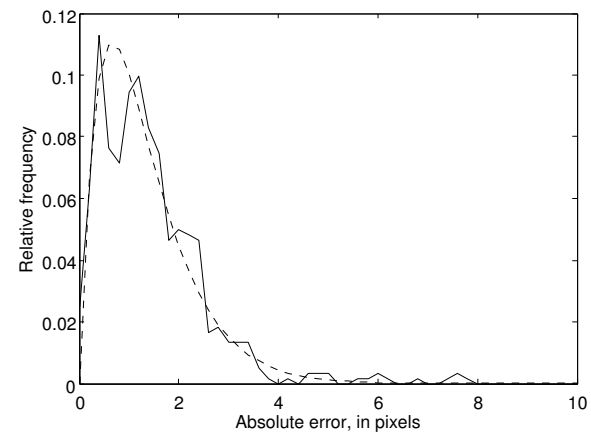

Figure 5. Bifurcation detection error (continuous line), in pixels. Dashed line curves are the best fit with $r e^{-r / a}$.

the detected bifurcation. This is intrinsic for the scale space methods; in strongly asymmetric bifurcations, the bifurcation is detected at the edge of the main branch instead of the center.

The algorithm was tested on clinical data as well. Only basic preprocessing was applied to the images - background removal (by subtracting a version of the image blurred by a wide Gaussian) and contrast stretching. We used 5 images, from large retinal angiograms, enhanced with fluorescein, each containing several hundred bifurcations. The original images had nonuniform background (approximated by a very large scale version of the image and subtracted). Figure 6(a) presents a small portion from such an image. With one exception, all the detected maxima were inside the vessels. Out of the 421 bifurcations present in one the images, 403 were detected, a detection rate of $95.7 \%$. All of the test images had detection rates between 95 and $97 \%$. A visual inspection of the bifurcations which were not detected showed that they have local maxima of are difficult cases, with one of the vessels coming out of the bifurcation having intensity close to the remaining background noise. These bifurcations still have local maxima of $\left|\lambda_{1}\right|$, but their

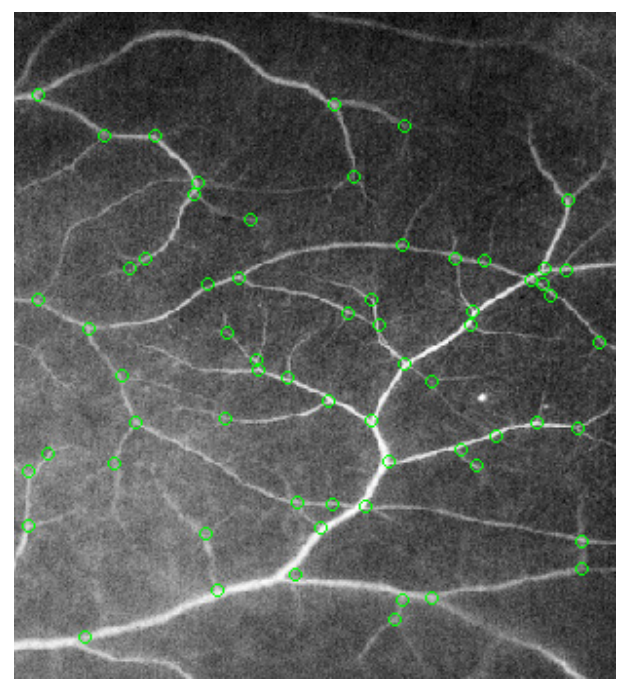

(a)

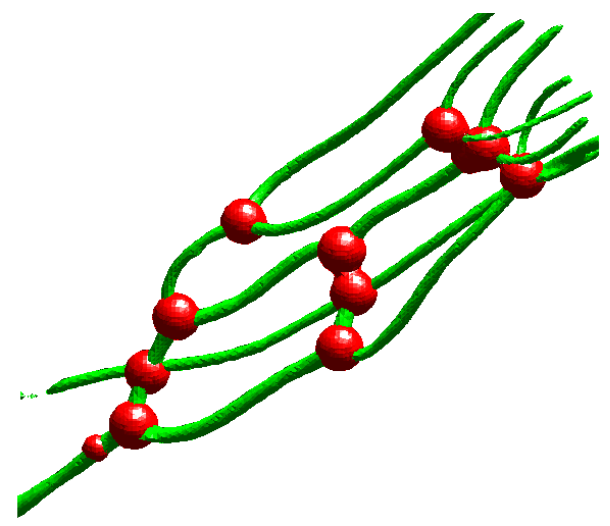

(b)

Figure 6. (a) Results of applying the bifurcation detection to a retinal image. Green circles mark the position of detected maxima, corresponding to potential bifurcations. (b) An illustration of detected bifurcations in 3D; the bifurcations (marked with blobs) were detected with the bifurcationness filter.

saliency and codimension were similar to those for noise. The codimension is a rather strong constraint, eliminating almost all of the false positives (only two were detected in the test images, probably due to elongated vessel thickening). The images also contained five blobs (two shown in Figure 6(a), correctly identified by the codimensionality criterion.

In 3D, the tests on synthetic bifurcations showed that the algorithm is able to discern bifurcations even at the lowest bifurcation ratio used (here we limited the minimum at 0.25 diameter ratio, or 0.06 area ratio, still below the 0.1 area ratio reported in literature). On a 3D phantom (nerve fibers in a block of wax), presented in Figure 6(b), all bifurcations and crossings were correctly identified. Only one false positive was identified (at a signal strength about $1 / 3$ of of sig- 
nal strength at bifurcations), located at the point where the nerve fiber enters the wax block.

\section{Conclusion and future directions}

In conclusion, we showed that it is possible to build a simple bifurcationness filter with good sensitivity, based on a simple scale-space conversion of the original image. Although simple, a complete scale-space analysis of the bifurcations was never done before. We showed that the smallest eigenvalue of the Hessian works well for detecting virtually all bifurcations in both $2 \mathrm{D}$ and $3 \mathrm{D}$, while a new filter, obtained by analyzing the structure of bifurcations, was proposed for 3D images. These simple combinations of eigenvalues work at least as well as the Frangi-style filters in 2D images, while they perform significantly better for 3D images. Our comprehensive analysis showed that the bifurcations have a hybrid structure, with spatial structures characteristic to both blobs and plates, and scale-space evolution characteristic to linear structures (vessels). For the first time, we pushed scale-space analysis one step further by using the rate of change across scales to gain additional insight on the investigated structures, encoded in the parameter called codimension. The accuracy of the detected bifurcation was good as long as no other features were in close proximity of the bifurcations, with most of the detected bifurcations within one vessel radius of the real position. Adding moderate noise did not affect significantly the accuracy of the algorithm, as scale-space is inherently immune to small amounts of noise. Even the salt-and-pepper noise has little influence if present in moderate amounts.

A main advantage of the proposed algorithm is that it is not intrinsically serial, and therefore can be very efficiently implemented on modern massively multicore processors.

The algorithm proposed can be improved, with further research. The location of bifurcation, as detected by the algorithm, can be further improved. In the case of strongly asymmetric bifurcations, the detected point is on the wall of the parent vessel, rather than its centerline. For more symmetric bifurcations, the detected point is on the extension of the parent vessel, between the daughter vessels. This is due to the gradual merging of features in scale-space [9]. The local spatial maximum of the bifurcation migrates with scale; we can detect this local maximum at two nearby scales and extrapolate its position to the scale $\sigma=0$ and obtain a better estimate of its position. However, this extension of the algorithm is needed only if extreme precision is required for localization, since the detected position is already within a (parent) vessel radius from its correct location.

The algorithm does not (and is not intended to) distinguish between bifurcations and crossings. They are put together by our detection algorithm, and left as candidates for other, more intensive algorithms. The simplicity of the al- gorithm - one-step, without requiring training data - makes it a good addition to the arsenal of medical diagnosis.

\section{References}

[1] D.-M. Baboiu. Vessel bifurcation detection in scale space. Master's thesis, Simon Fraser University, Canada, 2011. https://theses.lib.sfu.ca/thesis/etd6562.

[2] L. M. J. Florack, B. M. ter Haar Romeny, J. J. Koenderink, and M. A. Viergever. Scale and the differential structure of images. Image Vision Comput., 10(6):376-388, 1992.

[3] A. F. Frangi, W. J. Niessen, K. L. Vincken, and M. A. Viergever. Multiscale vessel enhancement filtering. In Medical Image Computing and Computer-Assisted Interventation - MICCAI'98, LNCS, page 130. Springer, 1998.

[4] Y. Fridman, S. M. Pizer, S. R. Aylward, and E. Bullitt. Segmenting $3 \mathrm{~d}$ branching tubular structures using cores. In $M I C$ CAI (2003, pages 570-577, 2003.

[5] Y.-C. Fung. Biomechanics: circulation. Springer, 2nd edition, 1997.

[6] C. Kirbas and F. Quek. A review of vessel extraction techniques and algorithms. ACM Comput. Surv., 36(2):81-121, 2004.

[7] J. J. Koenderink. The structure of images. Biological Cybernetics, 50(5):363-370, August 1984.

[8] D. Lesage, E. D. Angelini, I. Bloch, and G. Funka-Lea. A review of 3d vessel lumen segmentation techniques: Models, features and extraction schemes. Medical Image Analysis, August 2009.

[9] T. Lindeberg. Scale-Space Theory in Computer Vision. Kluwer Academic Publishers, Norwell, MA, USA, 1994.

[10] T. Lindeberg. Edge detection and ridge detection with automatic scale selection. In CVPR '96: Proceedings of the 1996 Conference on Computer Vision and Pattern Recognition (CVPR '96), page 465, Washington, DC, USA, 1996. IEEE Computer Society.

[11] M. Maddah, H. Soltanian-Zadeh, and A. Afzali-Kushaa. Snake modeling and distance transform approach to vascular centerline extraction and quantification. Computerized Medical Imaging and Graphics, 27(6):503-512, 2003.

[12] C. Mcintosh and G. Hamarneh. Vessel crawlers: 3d physically-based deformable organisms for vasculature segmentation and analysis. In Computer Vision and Pattern Recognition, 2006 IEEE Computer Society Conference on, volume 1, pages 1084-1091, 2006.

[13] T. Pock, R. Beichel, and H. Bischof. A novel robust tube detection filter for $3 \mathrm{~d}$ centerline extraction. In Proc Scandinavian Conference on Image Analysis (SCIA), volume 3540 of LNCS, pages 481-490. Springer, 2005.

[14] G. Szegö. Orthogonal Polynomials. American Mathematical Society, 1939, 1955.

[15] M. Zamir, S. Wrigley, and B. Langille. Arterial bifurcations in the cardiovascular system of a rat. Journal of General Physiology, 81:325-335, 1983.

[16] J. Zhou, S. Chang, D. Metaxas, and L. Axel. Vascular structure segmentation and bifurcation detection. In Biomedical Imaging: From Nano to Macro, ISBI 2007, pages 872-875, 2007. 